\title{
FATIGUE AND CORROSION FATIGUE LIFE ASSESSMENT WITH APPLICATION TO AUTOFRETTAGED PARTS
}

\author{
Volodymyr Okorokov \\ University of Strathclyde \\ Glasgow, the UK
}

\author{
Donald MacKenzie \\ University of Strathclyde \\ Glasgow, the UK
}

\author{
Yevgen Gorash \\ University of Strathclyde \\ Glasgow, the UK
}

\begin{abstract}
This study investigates an effect of autofrettage on the fatigue and corrosion fatigue life of high pressure parts made from low carbon structural steel. To estimate the beneficial effect of autofrettage application, an extensive experimental program and advanced theoretical modelling are conducted and analyzed in this study. Accurate calculation of compressive residual stresses is achieved by application of a cyclic plasticity model which can precisely simulate a cyclic plasticity response of material. In terms of a fatigue life prediction methodology, a non-local stress based approach with a modified critical distance theory is used for prediction of the crack initiation stage providing conservative fatigue assessment. Because of the fact that the crack propagation stage can take a considerable part of the total life for autofrettaged parts, more accurate fatigue life calculation is performed by the use of a fracture mechanics approach. The total fatigue life time of autofrettaged parts is then calculated as a sum of the crack initiation and propagation stages.
\end{abstract}

\section{NOMENCLATURE}

$p$ - accumulated plastic strain

i - accumulated plastic strain rate

$\therefore$ - previously accumulated plastic strain

$q$ - plastic strain amplitude

$i_{i}$ - previously accumulated plastic strain amplitude

$\varepsilon_{e q}^{p}-$ equivalent plastic strain

$\bar{\varepsilon}_{e q}^{p}-$ previously accumulated equivalent plastic strain

$\varepsilon_{i j}^{p}-$ plastic strain tensor

$i_{y}$ - plastic strain rate tensor

$\tau$ - time delay

$\sigma_{f}$ - fatigue limit at a given $\mathrm{R}$ ratio

$L$ - critical distance parameter $\sigma_{e q}^{a}$ - equivalent stress amplitude

$K_{\text {max }}-$ maximum stress intensity factor

$K_{o p}$ - opening stress intensity factor

$\Delta K_{t h}$ - fatigue crack propagation threshold

$a$ - crack length

$N$ - number of cycles during crack propagation

$C$ - material constant from Paris law

$m$ - material constant from Paris law

$Z$ - argument of the Dirac delta function

\section{INTRODUCTION}

Autofrettage is a well-known method of increasing high cycle fatigue life for a wide range of high pressure components working in dynamic conditions. The idea of the method is to induce a large magnitude of plastic strains by application of very high overloading pressure. With releasing the overload a large amount of compressive residual stresses occurs in highly stressed locations of a component. These compressive residual stresses reduce the mean value of stresses occurred after application of a working load, thereby leading to increase of the fatigue life.

There have been numerous studies [1 - 5] regarding to autofrettage modelling. Modelling concept is usually divided into calculation of compressive residual stress field after application of the autofrettage process and prediction of the fatigue lifetime under cyclic loading with the induced compressive residual stresses.

Accuracy of the compressive residual stresses calculation depends on the availability of material testing data with plasticity material response and the ability of a theoretical approach to simulate this plasticity behavior. There is a large number of cyclic plasticity models with different expressions of flow rules for kinematic and isotropic hardening available in literature [6 8]. However, when engineering problem requires only a few cycles of loading-reloading, as for the autofrettage and reautofrettage processes, these models might not give very accurate 
predictions. This is because constants of these models are usually calibrated to either monotonic or cyclic stress-strain curves. Therefore, these models are not able to accurately describe the first monotonic stress-strain curve and subsequent cyclic curves at the same time. Only a few model [9 and 10] with a specific set of parameters are able to give reasonably accurate prediction of compressive residual stresses. In spite of the accuracy of this approach, there are a few disadvantages of the models such as a large number of back stress decompositions and complicated theoretical framework which can create some complications when applied in real engineering applications. In this study, a new plasticity model with a new set of internal variables is proposed. The model can provide a high accuracy of modelling stress-strain curve shape with a minimum number of material constants.

In terms of the prediction strategy of the high cycle fatigue, it is convenient to split the total fatigue life into the stages of the crack initiation and propagation. The crack initiation time in metals is linked to the nucleation and propagation of microstructurally small cracks. Standard fracture mechanics approaches are not applicable in this case. Works [11 - 14] present developments of the theory of critical distance which can adopt the stress prediction methodology for prediction the crack initiation stage by introducing a critical distance parameter. The main idea of the method is to average calculated structure stresses over a process volume of material and compare these to stresses obtained after testing of uniaxial samples. To include the plasticity effects, a modified critical distance method is used here. For most components working in high cycle fatigue conditions, the initiation life is usually dominant. However, compressive residual stress field induced by the autofrettage process can make the propagation stage longer. It is proven experimentally by many studies that fatigue crack growth is retarded inside the compressive residual stress field. Papers [15 -17] report the crack arrest phenomena of autofrettaged samples. In this case, compressive residual stresses completely arrest the crack propagation. Moreover, when metal is subject to fatigue in a corrosion environment the crack propagation stage is a dominant mode in fatigue failure. To model the crack propagation phenomena the fracture mechanics approach is used.

In this study, the effect of autofrettage on the high cycle fatigue life is investigated through experimental testing of low carbon structural steel S355 with basic material properties shown in "table. 1". With obtaining the plasticity response and fatigue strength parameters of this steel, the autofrettage effect is analyzed using special geometry of notched samples.

\section{CYCLIC PLASTICITY}

Autofrettage is essentially based on the phenomenon of plastic deformation. Plastic overload of a high pressure part generates a large magnitude of compressive residual stress in the part. The knowledge of plastic material response is, therefore, a crucial factor in modelling of the compressive residual stresses induced by autofrettage.
In order to investigate the cyclic and monotonic plasticity behavior, monotonic and cyclic tests with different loading programs were conducted. The samples were made from low carbon steel with the following geometry parameters: total length $-140 \mathrm{~mm}$; gauge length $-25 \mathrm{~mm}$; grip section width -20 $\mathrm{mm}$; gauge section width $-12 \mathrm{~mm}$; thickness $-6 \mathrm{~mm}$. The testing was done with the use of a $250 \mathrm{kN}$ INSTRON servohydraulic testing machine under strain control with a total strain rate of $5 \cdot 10^{-4} \mathrm{~s}^{-1}$ for both monotonic and cyclic loading. The strain was measured by an extensometer with $10 \mathrm{~mm}$ in gauge length.

Table. 1.

\begin{tabular}{|c|c|c|c|}
\hline $\begin{array}{c}\text { Young's } \\
\text { modulus }\end{array}$ & $\begin{array}{c}\text { Yield } \\
\text { stress }\end{array}$ & $\begin{array}{c}\text { Ultimate } \\
\text { stress }\end{array}$ & Elongation \\
\hline $200 \mathrm{GPa}$ & $255 \mathrm{MPa}$ & $501 \mathrm{MPa}$ & $37.5 \%$ \\
\hline
\end{tabular}

"Figure 1." illustrates the results from a few tension compression uniaxial tests at the loading-unloading stages. This test is of a particular interest for the autofrettage methods as the test shows a realistic material response similar to that from the application of autofrettage. The graph shows such important cyclic plasticity phenomena as the Bauschinger effect and elastic properties degradation with accumulation of plastic strain. The first unloading deformation curves are significantly softer than the initial monotonic stress-strain curve due to the Bauschinger effect. However, subsequent cyclic loading will cause the cyclic hardening phenomenon. It should be noted that a proper modelling of these phenomena is not possible without an appropriate plasticity model.

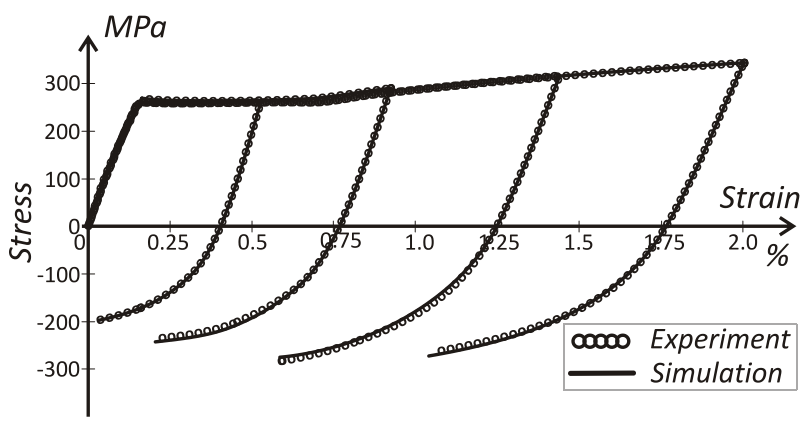

Figure 1. Cyclic plasticity material response.

Improved accuracy of modelling is achieved by developing a new set of internal variables which are used to incorporate the effects of strain range dependence and transition from the initial monotonic stress-strain curve to subsequent curves of cyclic loading. These effects are incorporated into constitutive modelling through introduction of a Dirac delta function with the following argument:

$$
Z=\frac{1}{2}\left[p-p^{\prime}-\operatorname{sign}\left(\varepsilon_{e q}^{p}-\varepsilon_{e q}^{\prime p}\right)\left(\varepsilon_{e q}^{p}-\varepsilon_{e q}^{\prime p}\right)\right]
$$

where $\varepsilon_{e q}^{p}, \bar{q}^{\prime}, \bar{p}^{\prime}, p^{\prime}$ and $\varepsilon_{e q}^{\prime p}$ are defined as follows: 


$$
\begin{aligned}
\varepsilon_{e q}^{p} & =\sqrt{\frac{2}{3} \varepsilon_{i j}^{p} \varepsilon_{i j}^{p}} \\
\bar{q}^{\prime} & =\bar{q}(t-\tau) \\
p^{\prime} & =p(t-\tau) \\
\bar{p}^{\prime} & =\bar{p}(t-\tau) \\
\varepsilon_{e q}^{\prime p} & =\varepsilon_{e q}^{p}(t-\tau)
\end{aligned}
$$

The main feature of this approach is an instant change of internal variables after changing of the flow direction. The Dirac function returns a required value at the beginning of a new step of loading with a change of the flow direction. At other moments of time the Dirac function returns zero value and the variables remain unchanged during the plastic deformation. This allows the variables to be constants on the current step of loading and change their value only at the beginning of a new step. Higher accuracy of cyclic hardening or softening can then be achieved by introducing strain range dependence into constants of the isotropic and kinematic hardening rules.

\section{RESIDUAL STRESS SIMULATION}

Fatigue testing of autofrettaged components in pressure test rigs requires specialist facilities and can be prohibitively expensive. The test methodology proposed and used here considers the behavior of a double notched tensile test specimen, as defined in "fig. 2.", in which a representative compressive residual stress field is induced through tensile overloading. A range of values of compressive residual stresses were induced in specimen notch region using the $250 \mathrm{kN}$ INSTRON machine.

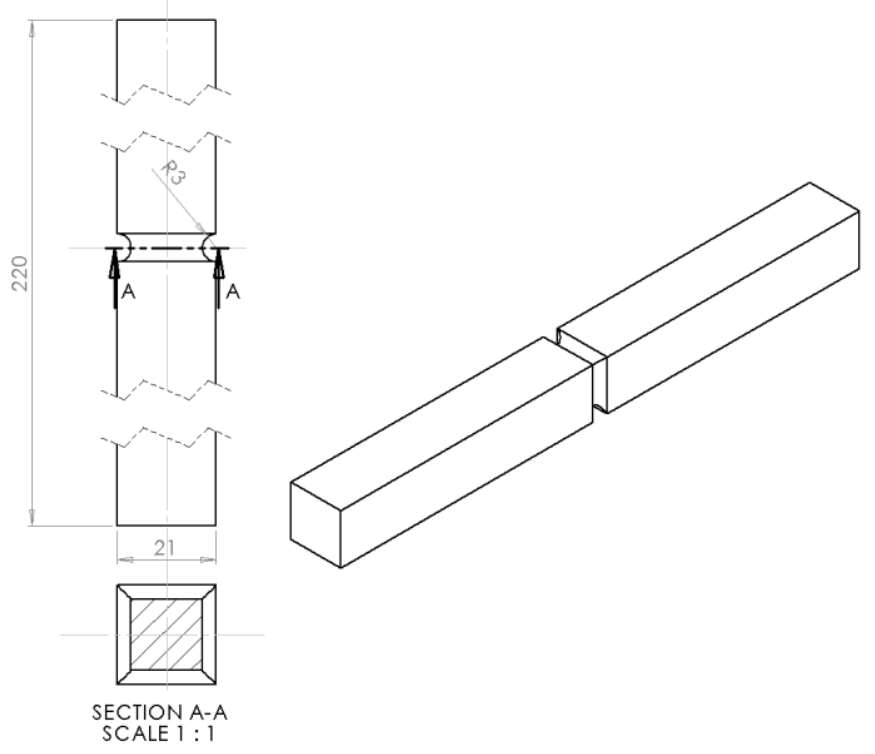

Figure 2. Double notched sample geometry.

The autofrettage process is simulated by applying overload remote (applied far from the notch area) force at the ends of the sample in order to induce a required value of plastic strain at the corner of intersected notches. With unloading of the sample a high magnitude of compressive residual stresses is generated within the notch root of the sample.

The numerical simulation is implemented by means of FEM with the use of ANSYS Workbench. The proposed plasticity model is incorporated into ANSYS Workbench by the means of User Programmable Features (UPF), where user implements custom equations and solving algorithms.

"Figure 3." shows results of the simulation of compressive residual stresses for an eighth part of the double notched sample. On the plots the compressive residual stress field is generated by overloading the sample with $75 \mathrm{kN}$ of remote tensile loading. The simulation is made by the use of the proposed model of plasticity and standard model with Chaboche nonlinear kinematic hardening which are embedded into ANSYS Workbench by default. The Chaboche nonlinear kinematic hardening model is calibrated to the initial monotonic stress-strain curve. The results show a fairly different distribution of the compressive residual stresses and a huge overestimation of the compressive residual stress magnitude with the use of the standard model. This happens because the standard models are not able to properly describe stress-strain curve for the unloading stage. The overestimation of the compressive residual stresses may mean that the fatigue life prediction can also be overestimated. This may lead to wrong design decisions with catastrophic effects.

It should be noted that the compressive residual stress field of the doubled notched samples is very similar to that from a real autofrettaged pressure part with a high stress concentration at the corner of intersected bores. Therefore, testing of that type of samples can give reasonable estimation of what should be expected with testing of the real pressure parts.
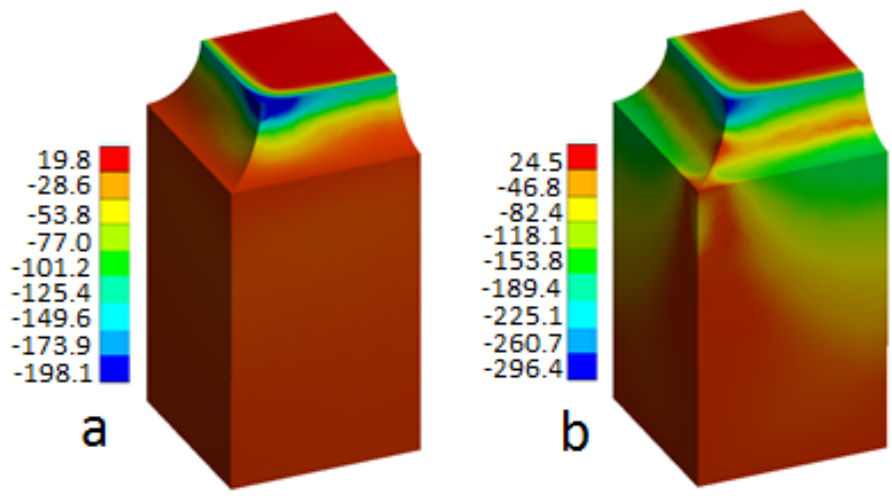

Figure 3. Compressive residual stress field (minimum principal stress) after unloading by a) proposed plasticity model; b) nonlinear Chaboche kinematic hardening.

\section{FATIGUE WITH AUTOFRETTAGE EFFECT}

The overloaded samples with the autofrettage effect are tested for the fatigue lifetime by force controlling tests. The loading ratio of the applied cyclic remote force was $\mathrm{R}=0$ for all the tests. The tests are performed in air and tap water environment. 


\section{Test results}

"Figure 4." illustrates experimental SN curves from fatigue testing in air. It is seen that the autofrettage effect increases the fatigue limit of the overloaded samples by $20 \%$ compared to the experimental results without overloading the sample. This relatively small increase in life is explained by the fact that for this type of low carbon steel the initial yield stress $(255 \mathrm{MPa})$ is less than the maximum stress $(370 \mathrm{MPa})$ at the fatigue limit for $R=0$ loading ratio. That means, in order to have failure of the double notched samples with $R=0$ force ratio, the yield point should be exceeded in the first cycle of loading following the plastic shakedown effect. This unavoidably leads to inducing compressive residual stresses in the notch root of the sample. This phenomenon is usually referred as the elastic and plastic shakedowns. For such type of material in fatigue loading conditions the effect of autofrettage is present even without overloading.

"Figure. 5." illustrates results of corrosion fatigue testing with $10 \mathrm{~Hz}$ of loading frequency. Corrosive environment is provided by testing samples in a chamber with tap water supply. S355 steel is subject to an intensive corrosion chemical reaction even in a tap water environment. Therefore, the fatigue lifetime is substantially reduced by the corrosion effect. The corrosion fatigue testing results presented in "fig. 5." show that overloading the double notched samples has a great improvement of the fatigue life. Given the fact that in a corrosion environment the fatigue limit does not exist or might exist at a very small level of stresses, the fatigue life improvement of the overloaded samples in region of $10^{8}-10^{9}$ number of cycles is even higher. This is because the difference of the slope angle of the overloaded and non-overloaded samples. It should also be noted that the difference between the air and corrosion fatigue results for the notched samples is not that significant as for the case of plain samples tested in uniaxial conditions. This may be explained by the fact that in the case of the notched sample if a crack is initiated at the notch root of the sample at the same stress level as for the uniaxial sample, the crack is significantly retarded with propagation because of the stress gradient and compressive residual stress field.

\section{Stress based prediction}

The numerical calculations are performed with the use of the conventional stress based assessment procedure, critical distance theory method and fracture mechanics approach.

According to the conventional stress based method, calculated stresses at a material point on the surface are directly compared to stresses obtained from uniaxial testing. For the case of the double notched sample the maximum alternating stresses are found at the notch roots of the samples. It is seen that prediction made by comparing these stresses directly to those from the uniaxial testing underestimate the fatigue life time. This is explained by the presence of a high stress gradient in the notched area, so that the stresses rapidly decrease moving away from the stress concentration. In the situation with high stress concentrations the fatigue life should be estimated by calculating stresses in a fatigue process volume of material rather than a material point at the surface of a component.

This is done by the use of the critical distance method in which calculated stresses are averaged over some distance from the component surface. There are several option for this method in which stresses are averaged over either line, area or volume. In the simplest option the critical distance is determined with the use of fracture mechanics approach as follows:

$$
L=\frac{1}{\pi}\left(\frac{\Delta K_{t h}}{\sigma_{f}}\right)^{2}
$$

The prediction of the fatigue limit of a component with stress concentration is then made according to:

$$
\sigma_{e q}^{a}(L / 2)=\sigma_{f}
$$

The attractiveness of the method is conditioned by the fact that a reasonable accuracy is reached by only post processing results of FEM calculation with the use of only few parameters. However, there is a limitation which make this method not applicable to the case of estimation of the fatigue life of components with residual stress field. The inability is explained by the fact that all quantities of the methods are derived or calibrated based on a constant R-ratio which represents the loading ratio of the applied load. In the case of cyclic loading with induced residual stresses the R-ratio of stresses is not constant. The modified critical distance method is used here. It is based on an iteration procedure that can give an average value of R-ratio acting in a considered area of a component.

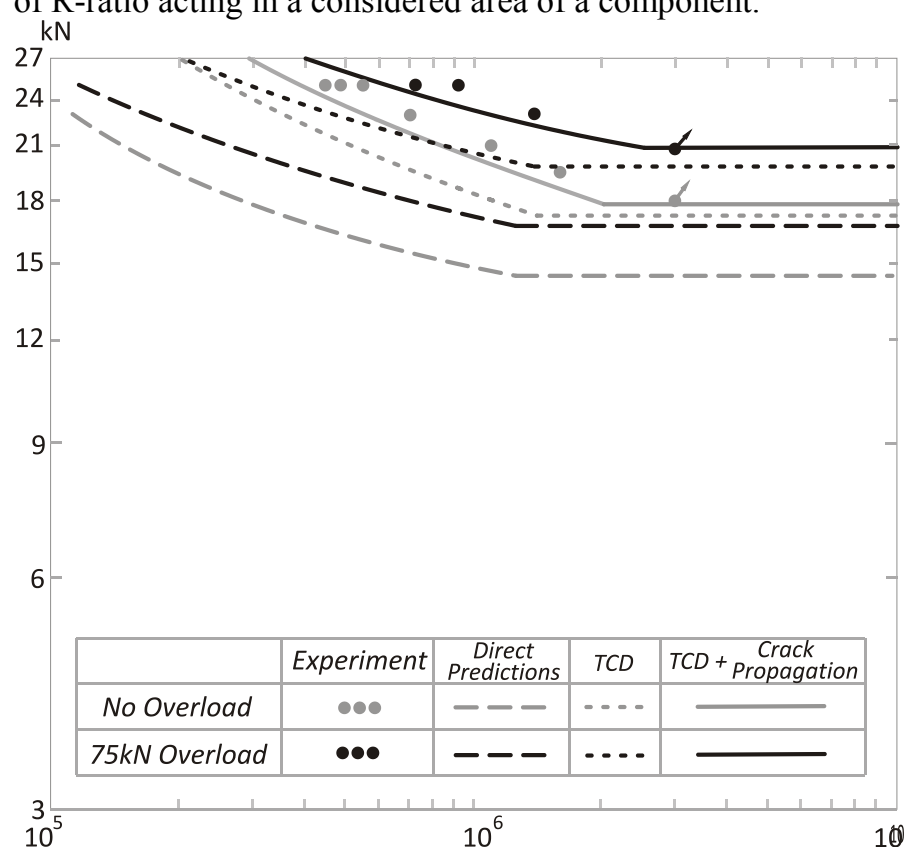

Figure 4. Experimental and numerical results of the fatigue testing in air.

It is seen from "fig. 4." that with the use of the critical distance method predictions of the fatigue life in air are much closer to 
the experimental results. In general, stress based assessment methods predict the crack initiation life. Close prediction of stress based methods for fatigue in air means that the crack initiation life appeared to be much longer than the crack propagation life.

In the case of corrosion fatigue testing numerical results obtained by the stress based assessment methodology are far from the experimental results from "fig. 5.". Critical distance correction makes prediction closer to experimental results but the difference is still considerable. This is because calculations made by this methodology actually predict the crack initiation life. The rest of the life is the crack propagation stage which is dominant in the corrosion fatigue life of both overloaded and non-overloaded samples.

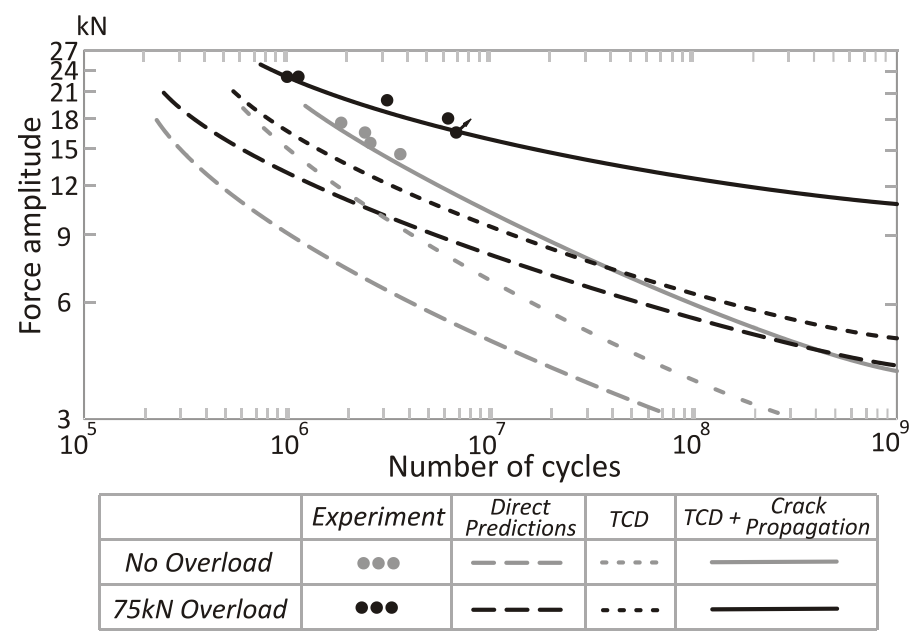

Figure 5. Experimental results and numerical predictions of the fatigue testing in water.

\section{Fracture mechanics prediction}

In order to calculate the total fatigue life which consists of the crack initiation and crack propagation stages application of fracture mechanics approaches is required. In this study the concept of crack closure is used for defining a driving force for crack propagation. According to this the effective stress intensity factor range is defined as:

$$
\Delta K_{\text {eff }}=K_{\text {max }}-K_{o p}
$$

Crack propagates according to the Paris law:

$$
\frac{d a}{d N}=C\left(\Delta K_{e f f}\right)^{m}
$$

For the case of fatigue in air, a crack propagates with fulfilling the following condition:

$$
\Delta K_{\text {eff }}>\Delta K_{t h}
$$

That condition essentially means that if the effective stress intensity factor range is less than the effective crack propagation threshold a crack is arrested and this corresponds to the fatigue limit on the SN curve. Material constants for the crack propagation law are found in [18].
With regards to fatigue in a corrosion environment it has been found that there is no fatigue limit on the SN curve [19] and the crack propagation threshold is significantly less compared to the one in air fatigue conditions. Therefore, in this study the crack propagation simulation for corrosion fatigue is performed with the assumption that there is no crack propagation threshold.

It is seen from "fig. 5." that prediction made as a sum of cycles predicted by the theory of critical distance and crack propagation simulation is very close to the experimental results. In this separation the critical distances predictions essentially mean the number of cycles to reach a given value of initial crack size where fracture mechanics is applicable to predict the rest of the fatigue life.

\section{CONCLUSIONS}

This paper presents the results of experimental investigation and numerical calculations of the fatigue and corrosion fatigue lives of low carbon steel with the autofrettage effect. The geometry of the double notched sample used in the study provides a similar 3D stress-strain distribution to a real pressure part, such that the effects expected from testing a real autofrettaged pressure part are observable in a simpler type of test specimen. This can provide estimation of the autofrettage effect at an early stage in the design and validation processes.

To calculate the fatigue lifetime of components with the autofrettage compressive residual stresses the following steps are proposed:

1. Calculation of the residual stress field which occurs after application of autofrettage;

2. Calculation of redistribution of the residual stress field due to plasticity effects occurred in the initial number of cycles before cyclic stabilization during the fatigue life;

3. Calculation of a number of cycles to the initiation of cracks in highly stresses locations of the structure by the use of stress based prediction methodology with a critical distance correction.

4. Calculation of the remaining number of cycles during the crack propagation stage with the use of the fracture mechanics approach.

The future research work includes experimental investigation of the fatigue and corrosion fatigue of full-scale autofrettaged pressure parts with application of the developed fatigue and corrosion fatigue lives prediction methodology.

\section{ACKNOWLEDGMENTS}

This project has received funding from the European Union's Horizon 2020 research and innovation programme under the Marie Sklodowska-Currie grant agreement No 643159.

\section{REFERENCES}

1. Adibi-Asl R. and Livieri P., 2007, "Analytical Approach in Autofrettaged Spherical Pressure Vessels Considering the Bauschinger Effect," J. Pressure Vessel Technology, Vol. 129 , pp. 411-419. 
2. Wahi N., Ayob A. and Elbasheer M. K., 2007, "Effect of Optimum Autofrettage on Pressure Limits of Thick-Walled Cylinder," Int. J. Environmental Science and Development, Vol.2, pp. 329-333.

3. Trojnacki A. and Krasiński M., 2014, "Numerical Verification of Analytical Solution for Autofrettaged Highpressure Vessels," J. Theoretical and Applied Mechanics, Vol. 52, pp. 731-744.

4. Jahed H., Farshi B. and Hosseini M., 2006, "Fatigue life prediction of autofrettage tubes using actual material behavior," Int. J. Pressure Vessels and Piping, Vol. 83, pp. 749-755.

5. Parker A. and Underwood J., 1999, "Influence of Bauschinger Effect on Residual Stress and Fatigue Lifetimes in Autofrettaged Thick-Walled Cylinders," Fatigue and Fracture Mechanics, Vol. 29. Pp. 565-583.

6. Lee C.H., Do V.N.V and Chang K.H., 2014, "Analysis of uniaxial ratcheting behavior and cyclic mean stress relaxation of a duplex stainless steel," International Journal of Plasticity, Vol. 62, pp. 17-33.

7. Xu L., Nie X., Fan J., Tao M. and Ding R., 2016, “Cyclic hardening and softening behavior of the low yield point steel BLY160: Experimental response and constitutive modeling," International Journal of Plasticity, Vol. 78, pp. 44-63.

8. Feigenbaum H.P., Dugdale J., Dafalias Y.F., Kourousis K.I. and Plesek J., 2012, "Multiaxial ratcheting with advanced kinematic and directional distortional hardening rules," International Journal of Solids and Structures, Vol. 49, pp. 3063-3076.

9. Döring R., Hoffmeyer J., Seeger T. and Vormwald M., 2003, "A plasticity model for calculating stress-strain sequences under multiaxial nonproportional cyclic loading," Computational Materials Science, Vol. 28, pp. 587-596.

10. Voyiadjis G.Z., Hoseini S.H. and Farrahi G.H., 2012, “A Plasticity Model for Metals With Dependency on All the Stress Invariants," Journal of Engineering Materials and Technology, Vol. 135, 011002-011002-011013.

11. Taylor D., 2008, "The theory of critical distances," Engineering Fracture Mechanics, Vol. 75, pp. 1696-1705.

12. Taylor D., Bologna P. and Bel K.K., 2000, "Prediction of fatigue failure location on a component using a critical distance method," International Journal of Fatigue, Vol. 22, pp. 735-742.

13. Susmel L., 2008, "The theory of critical distances: a review of its applications in fatigue," Engineering Fracture Mechanics, Vol. 75, pp. 1706-1724.

14. Susmel L. and Taylor D., 2006, "A simplified approach to apply the theory of critical distances to notched components under torsional fatigue loading," International Journal of Fatigue, Vol. 28, pp. 417-430.

15. Herz E., Hertel O. and Vormwald M., 2011, "Numerical simulation of plasticity induced fatigue crack opening and closure for autofrettaged intersecting holes," Engineering Fracture Mechanics, Vol. 78, pp. 559-572.

16. Herz E., Thumser R., Bergmann J.W. and Vormwald M., 2006, "Endurance limit of autofrettaged Diesel-engine injection tubes with defects," Engineering Fracture Mechanics, Vol. 73, pp. 3-21.

17. Sellen S., Maas S., Andreas T., Plapper P., Zürbes A. and Becker D., 2016, "Design rules for autofrettage of an aluminium valve body," Fatigue \& Fracture of Engineering Materials \& Structures, Vol. 39, pp. 68-78.
18. Rudd W. and Shuter D., 1997, "Studies of the mechanism of history effects in fatigue and corrosion fatigue," Technical Report EUR 13960 EN, European Commission, British Steel, Swinden Technology Centre.

19. Ragab A., Alawi H. and Sorein K., 1989, "Corrosion fatigue of steel in various aqueous environments," Fatigue \& Fracture of Engineering Materials \& Structures, Vol. 12, pp. 469-479. 